\title{
APLICAÇÕES DA TRIGONOMETRIA ESFERICA EM PROBLEMAS DA SIMETRIA CRISTALOGRÁFICA
}

\author{
William G.R. De Camargo \\ (da Universidade de São Paulc)
}

\begin{abstract}
The present article ceals with the application of elementary spherical trigonometry to solution of symmetry problems. The main purpose of the article is to point out symmetry axis associations, and to show the porsibilities and impossibilities of several associations, revealed through spherical trigonometric relations.

The following axis associations have been a matter of study:

a) $6+6$; b) $4+4$; c) $2+2$; d) $6+3$; e) $6+4$; f) $6+2$; g) $4+3$

For solution of all the proposed problems only one trigonometric formula has been used: $\cos a=\cos b \cos c+\operatorname{sen} b \operatorname{sen} c \cos A$

which is applicable to scalene triangles.
\end{abstract}

Em trabalho anterior (1) foi estudada a combinação de eixos ternários nos cristais, tendo sido discutido como essa combinação poderia ser resolvida com relativa facilidade por intermédio da trigonometria esférica.

No presente artigo serão tratadas associações de eixos de simetria de outras ordens, recorrendo ao mesmo método trigonométrico. Parte-se de uma dada hipótese, e se esta for incompativel, isto será revelado atravéz das deduções matemáticas, pelo aparecimento de resultados absurdos ou imaginários.

Os seguintes casos de associações serão objeto de análise no presente artigo: a) $6+6$; b) $4+4$; c) $2+2$; d) $6+3$ e) $6+4$; f) $6+2 ; g) 4+3$.

a) Associação $6+6$ - Supondo-se um eixo senário vertical e a êle associado um segundo eixo da mesma ordem, fazendo com o primeiro um certo ângulo $x$, desconhecido, o segundo eixo deve ser repetido seis wezes em um giro de $360^{\circ}$, em virtude da lei de recobrimento. O ângulo de recobrimento é igual a $60^{\circ}$ e igual também ao ângulo interno do triângulo esférico formado por três eisos senários vizinhos (fig. 1). 
Pela fig. 1, aplicando a fórmula geral da trigonometria esférica para triângulos escalenos, ter-se-á:

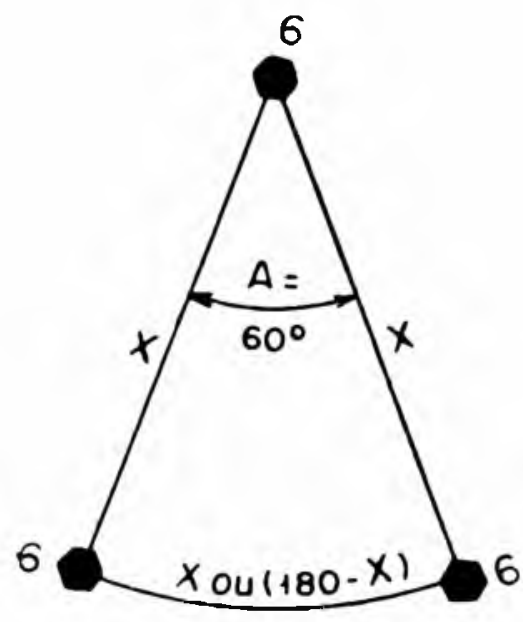

Fig. 1

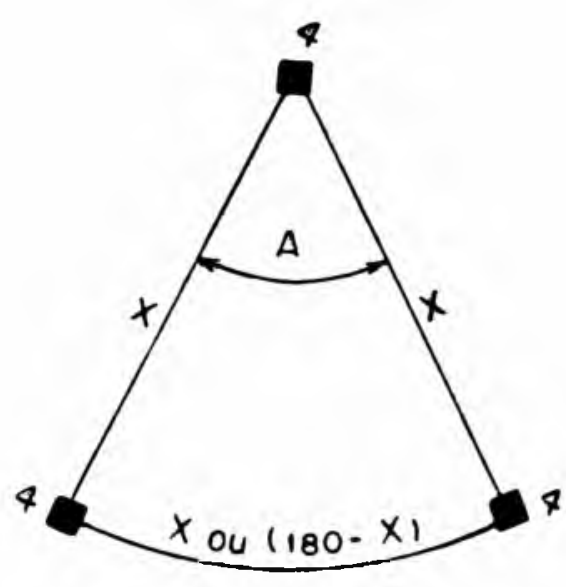

Fig. 2

$\cos a=\cos b \cos c+\operatorname{sen} b \operatorname{sen} c \cos A$

$\mathrm{b}=\mathrm{c}=\mathbf{x}$

$a=x$ ou $\left(180^{\circ}-x\right)$

$\mathrm{A}=60^{\circ}$

Por substituição:

$\pm \cos x=\cos ^{2} x+\operatorname{sen}^{2} x \cos 60^{\circ}$

$\pm \cos x=\cos ^{2} x+1 / 2 \operatorname{sen}^{2} x$

$\pm \cos x=\cos ^{2} x+1 / 2\left(1-\cos ^{2} x\right)$

$$
\cos ^{2} x \pm 2 \cos x+1=0
$$

Resolvendo esta equação trigonométrica (2) do segundo grau:

$$
\begin{gathered}
\cos x=\frac{ \pm 2 \pm \sqrt{4-4}}{2}=+1 \text { ou }-1 \\
x=O^{\circ} \text { ou } 180^{\circ}
\end{gathered}
$$

Conclue-se, por conseguinte, pela existência de um único 6 e qualquer associação entre eixos senários é absurda.

b) Associação $4+4-$ No caso dos eixos quaternários será feita a mesma suposição inicial imaginada para os eixos senários. Assim, por aplicação da fórmula (1) (fig. 2): 


$$
\begin{aligned}
& \pm \cos x=\cos ^{2} x+\operatorname{sen}^{2} \cos 90^{\circ} \\
& \pm \cos x=\cos ^{2} x \\
& \cos ^{2} x \pm \cos x=0
\end{aligned}
$$

Resolvendo esta equação (3):

$$
\begin{aligned}
\cos \mathrm{x} & =+1,0,-1 \\
\mathrm{x} & =0^{\circ}, 90^{\circ}, 180^{\circ} .
\end{aligned}
$$

Nos resultados $0^{\circ}$ e $180^{\circ}$, haverá existência de um único 4 , polar no primeiro caso e bipolar no segundo. Para $\mathrm{x}=90^{\circ}$, aparecem três eixos quaternários bipolares, em conseqüência do recobrimento tetrassimétrico (fig. 3)
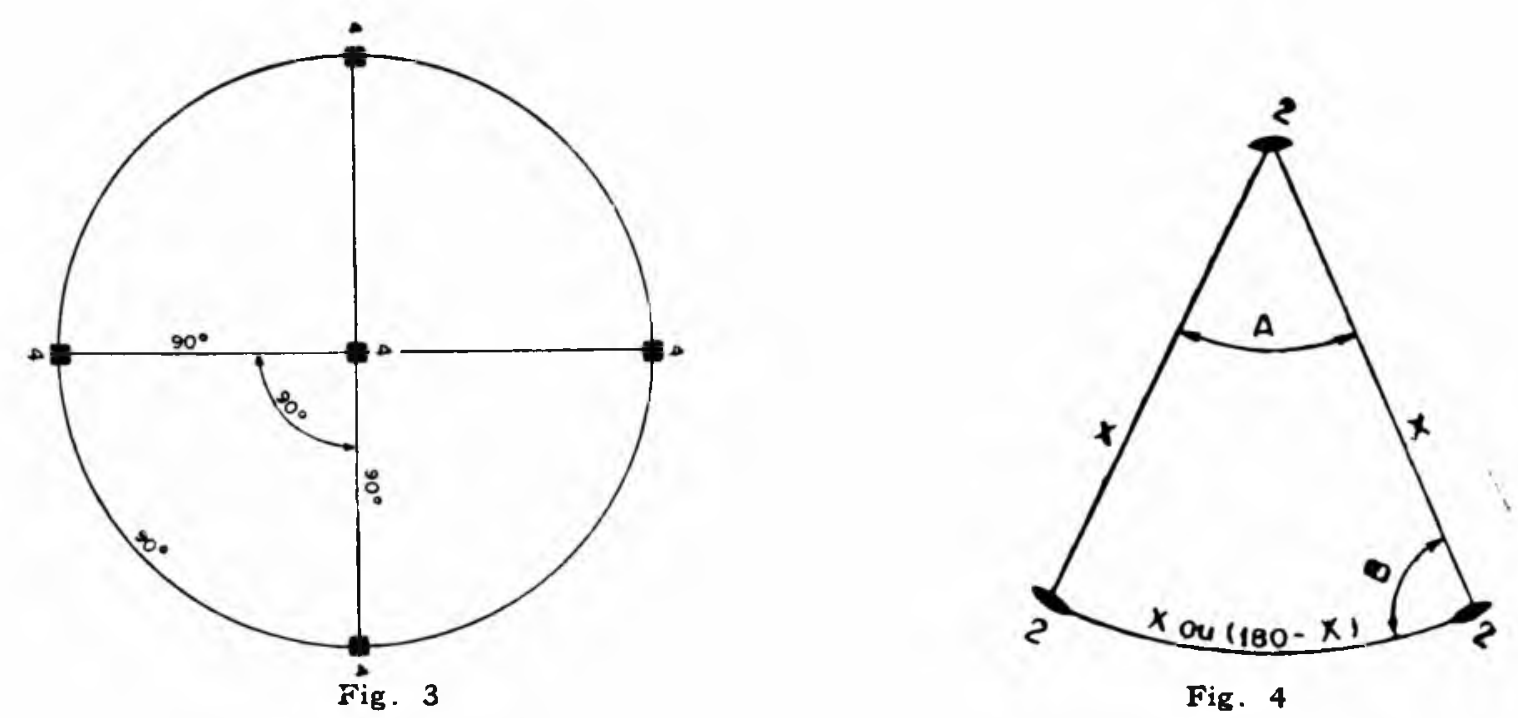

c) Associação $2+2$ - Considerando a fig. 4 e aplicando-se a fórmula (1) novamente:

$$
\begin{aligned}
& \pm \cos x=\cos ^{2} x+\operatorname{sen}^{2} \cos 180^{\circ} \\
& \pm \cos x=\cos ^{2} x-\operatorname{sen}^{2} x \\
& 2 \cos ^{2} x \pm \cos x-1=0 \\
& \text { Resolvendo esta equação }(4): \\
& \cos x=+1,-1,+1 / 2,-1 / 2 \\
& \quad x=0^{\circ}, 180^{\circ}, 60^{\circ}, 120^{\circ}
\end{aligned}
$$

Nos dois primeiros casos deve aparecer um único 2, polar e bipolar respectivamente. Quando $x=60^{\circ}$, dois casos são possíveis: 1- sistema hexagonal, nas classes onde ocorrem seis eixos binários (três de uma espé- 
cie e três de outra) (fig. 5); 2- sistema monométrico, nas classes que possuem seis 2 equivalentes, (fig. 6) Para $x=120^{\circ}$, o caso representa $o$ sistema trigonal, quando ocorrem três 2 polares (fig. 7)

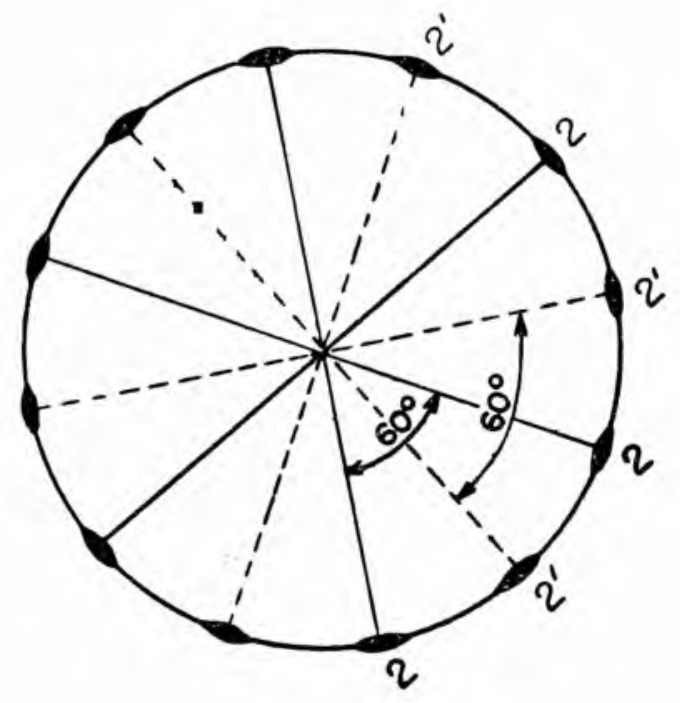

Fig. 5

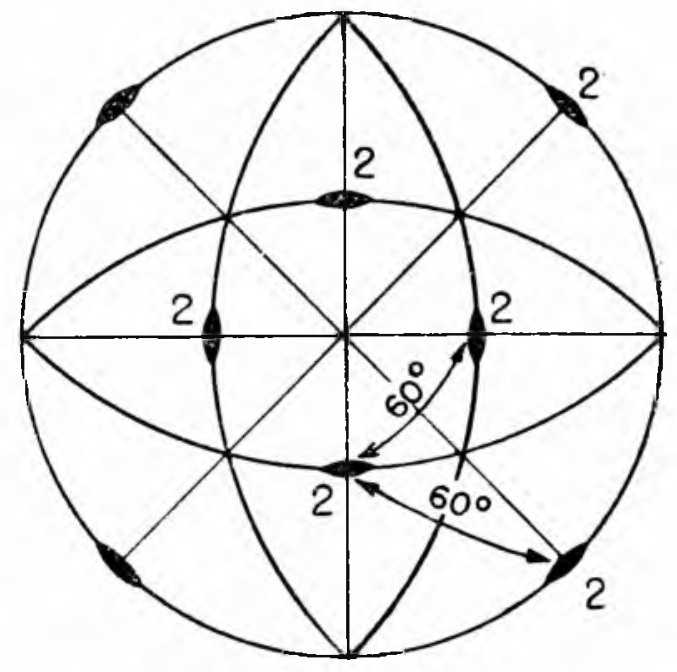

Fig. 6

Existem ainda classes de simetria, onde aparecem eixos binários ortogonais. São certas classes de simetria do sistema monométrico (da pirita com grao de simetria $\mathrm{m} 3$ e da ullmanita com grao de simetria 23) e algumas classes do sistema rômbico (da barita, $\mathrm{mmm}$, e da epsomita, 222 ).

Para provar a ortogonalidade dos eixos binárics nestes casos, deverá ser seguido caminho inverso daquele utilizado até agora. Antes conhecia-se $A$ e procurava-se x; agora será admitido um valor para x e $A$ será calculado. Pela simetria, verifica-se posteriormente a viabilidade da hipótese. Considerando a fig. 4:

$$
\begin{aligned}
& \cos a=\cos b \cos c+\operatorname{sen} b \operatorname{sen} c \cos A \\
& a=x \text { ou }\left(180^{\circ}-x\right) \\
& b=c=x=90^{\circ} \\
& \cos \mathbf{x}=\cos ^{2} x+\operatorname{sen}^{2} \mathbf{x} \cos A \\
& \cos 90^{\circ}=\cos ^{2} 90^{\circ} \cos A \\
& \cos \mathbf{A}=0 \\
& \mathbf{A}=90^{\circ}
\end{aligned}
$$


O ângulo A entretanto, não deve aqui ser considerado como período do eixo binário, que continua e deve ser $180^{\circ}$ A deve constituir apenas o ângulo interno do triângulo esférico, formado por três eixos binários imediatamente vizinhos. No sistema rômbico, os três eixos são de espécies diferentes, e no sistema monométrico, embora os três 2 sejam equivalentes e da mesma espécie, a configuração completa do grao de simetria, o qual ainda pode envolver a presença de eixos ternários polares, assegura o período de $180^{\circ}$ para os eixos binárins (fig. 8 e 9 ).

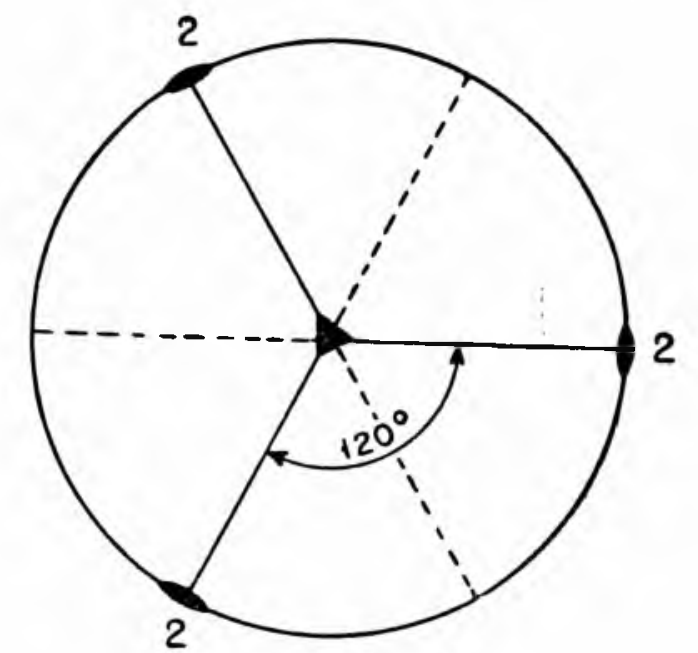

Fig. 7

d) Associação $6+3-0$ ângulo entre dois eixos ternários, como já foi deduzido em artigo anterior (1):

$$
\mathrm{y}=\mathrm{O}^{\circ}, 180^{\circ}, 109^{\circ} 28^{\prime} 16^{\prime \prime}
$$

Aplicando a fórmula (1) ao triângulo da fig. 10:

$$
\cos y=\cos ^{2} x+1 / 2 \operatorname{sen}^{2} x
$$

porque $A=60^{\circ}$ e $\cos 60^{\circ}=1 / 2$

$$
\begin{aligned}
& \cos y=\cos ^{2} x+1 / 2\left(1-\cos ^{2} x\right) \\
& \cos ^{2} x=2 \cos y-1 \\
& \cos ^{2} x+1=2 \cos y
\end{aligned}
$$

Dando valôres sucessivos a y $\left(\mathrm{O}^{\circ}, 180^{\circ}, 109^{\circ} 28^{\prime} 16^{\prime \prime}\right)$, teremos:

$$
\begin{aligned}
& \cos ^{2} x+1=2 \cos 0^{\circ} \\
& \cos x= \pm 1 \quad x=O^{\circ} \text { ou } 180^{\circ} \\
& \cos ^{2} x+1=2 \cos 180^{\circ} \\
& \cos ^{2} x=-3 \\
& \cos x=\text { imaginário }
\end{aligned}
$$




$$
\begin{aligned}
& \cos ^{2} x+1=2 \cos 109^{\circ} 28^{\prime} 16^{\prime \prime} \\
& \cos ^{2} x+1=2-0,3334 \\
& \cos ^{2} x=-1,6668 \\
& \cos x=\text { imaginário }
\end{aligned}
$$

No primeiro caso $\left(x=0^{\circ}\right.$ ou $\left.180^{\circ}\right), 6$ deve coincidir com 3, hipótese esta redundante, pois o eixo senário pode funcionar como ternário. As duas outras hipóteses são absurdas, em virtude dos resultados imaginá-

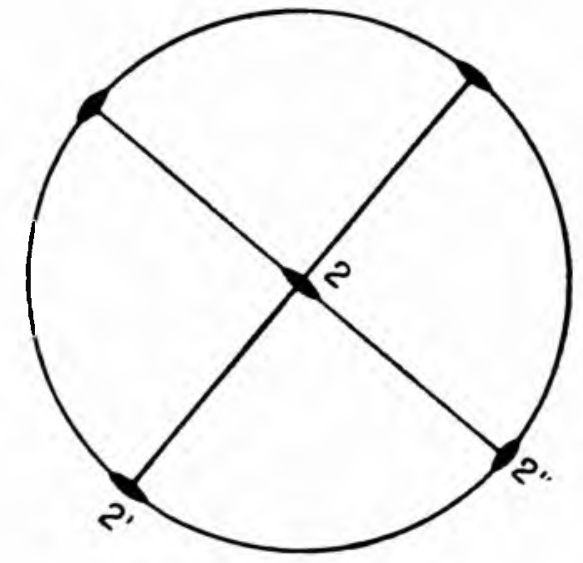

Fig. 8

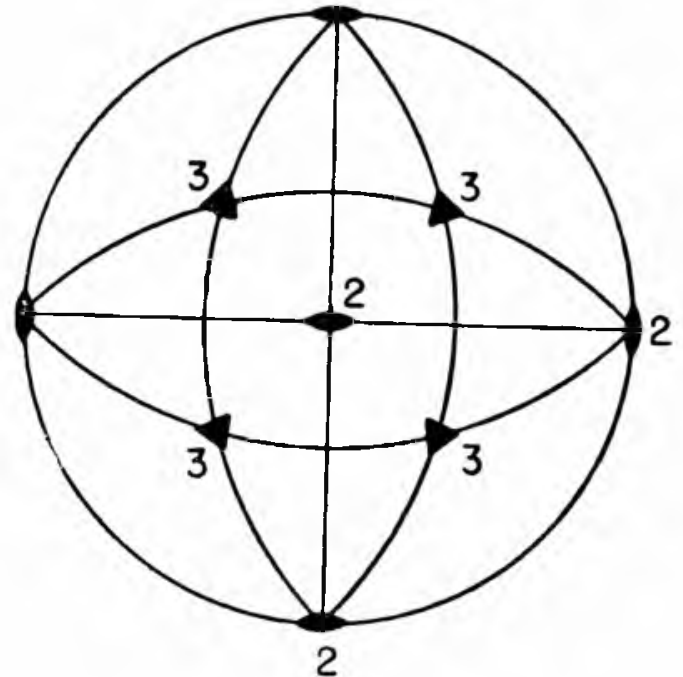

Fig. 9

rios. A conclusão final é pela impossibilidade dentro da simetria cristalográfica da associação de eið^o senário com ternário.

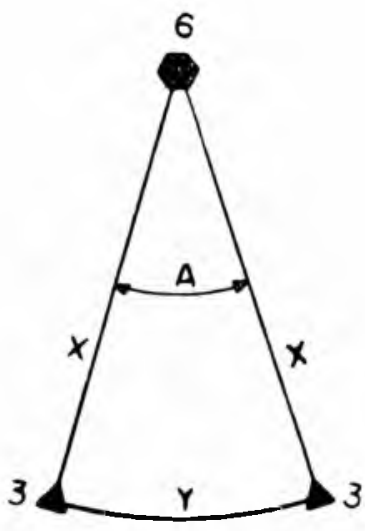

Fig. 10

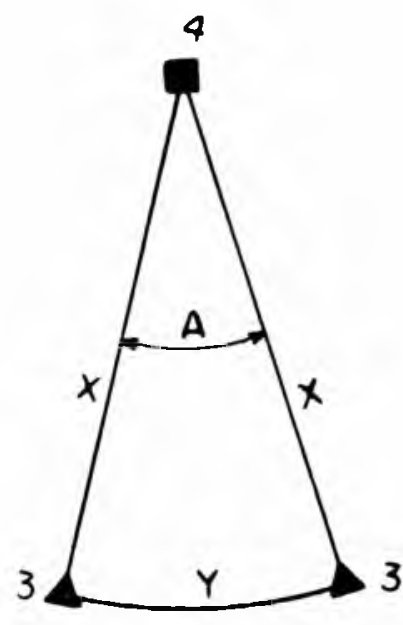

Fig. 11

e) Associação $6+4$ - Por nova aplicação da fórmula (1): $\cos y=\cos ^{2} \mathrm{x}+\operatorname{sen}^{2} \mathrm{x} \cos \mathbf{A}$ $\cos y=\cos ^{2} x+1 / 2 \operatorname{sen}^{2} x$ 


$$
\begin{aligned}
& \cos y=\cos ^{2} x+1 / 2\left(1-\cos ^{2} x\right) \\
& 2 \cos y=\cos ^{2} x+1
\end{aligned}
$$

Os valores possíveis de y, ângulo $4-4$ são $\mathrm{O}^{\circ}, 180^{\circ}$, e $90^{\circ}$. Substituindo êstes valores na equação (5):

$$
\begin{aligned}
& 2 \cos 0^{\circ}=\cos ^{2} x+1 \\
& \cos ^{2} x=1 \quad x=0^{\circ} \text { ou } 180^{\circ} \\
& \cos x= \pm 1 \quad x \quad \cos ^{2} x+1 \\
& 2 \cos 180^{\circ}=\cos ^{2} x=-3 \\
& \cos x=1 \\
& \cos x=\text { imaginário } \\
& 2 \cos 90^{\circ}=\cos ^{2} x+1 \\
& \cos ^{2} x=-1 \\
& \cos x=\text { imaginário }
\end{aligned}
$$

Nos três casos os resultados são absurdos. No primeiro por incompatilidade, pois um eixo senário não pode ser ao mesmo tempo um quaternário, e nos demais pelos resultados imaginários.

f) Associação $6+2-$ Foi visto anteriormente que o ângulo $\mathrm{y}$, entre dois eixos binários poderia assumir os seguintes valores: $0^{\circ}, 60^{\circ}$ $90^{\circ}, 120^{\circ}$ e $180^{\circ}$ Por aplicação da fórmula (1):

$$
\begin{aligned}
& \cos y=\cos ^{2} x+1 / 2 \operatorname{sen}^{2} x \\
& \cos ^{2} x=2 \cos y-1
\end{aligned}
$$

Substituindo os valores possíveis de y nesta equação (6):

$$
\begin{aligned}
& \cos ^{2} x=2 \cos 0^{\circ}-1 \\
& \cos ^{2} x=1 \\
& \cos x= \pm 1 \quad x=0^{\circ} \text { ou } 180^{\circ} \\
& \cos ^{2} x=2 \cos 60^{\circ}-1 \\
& \cos ^{2} x=2 \cdot 1 / 2-1 \\
& \cos x=0 \quad x=90^{\circ} \\
& \cos ^{2} x=2 \cos 90^{\circ}-1 \\
& \cos ^{2} x=-1 \\
& \cos ^{2} x=\text { imaginário } \\
& \cos ^{2} x=2 \cos 120^{\circ}-1 \\
& \cos ^{2} x=-2 \cdot 1 / 2-1 \\
& \cos ^{2} x=-2 \\
& \cos ^{2} x=- \text { imaginário } \\
& \cos ^{2} x=2 \cos 180^{\circ}-1 \\
& \cos ^{2} x=-3 \\
& \cos ^{2} x=\text { imaginário }
\end{aligned}
$$


Em tôdas as substituições, sòmente as duas primeiras são viáveis. No primeiro caso deveria haver coincidência de $6 \mathrm{com} \mathrm{2,} \mathrm{o} \mathrm{que} \mathrm{constitui}$ uma redundância, pois o eixo senário pode funcionar como binário. No segundo caso, o ângulo 6-2 deve ser igual a $90^{\circ}$, fato que sucede no sistema hexagonal.

g) Associação $4+3$ - Considerando a fig. 11, o ângulo 3-3 (y) apenas pode assumir os seguintes valores: $\mathrm{O}^{\circ}, 180^{\circ}$, e $109^{\circ} 2816^{\prime \prime}$ O ângulo interno $A$ é igual a $90^{\circ}$, em virtude do período do eixo 4 . Aplicandouse a fórmula (1):

上 $\cos y=\cos ^{2} x+\operatorname{sen}^{2} x \cos A$

$\pm \cos \mathrm{y}=\cos ^{2} \mathrm{x}$

Substituindo os valores possíveis de y nesta última equação:

$\pm \cos \mathrm{O}^{\circ}=\cos ^{2} \mathrm{x}$ $\cos ^{2} x= \pm 1$ $\cos ^{2} \mathrm{x}=+1 \quad \mathrm{x}=\mathrm{O}^{\circ}$ $\cos ^{2} \mathrm{x}=-1 \quad \mathrm{x}=$ imaginário

$\pm \cos 180^{\circ}=\cos ^{2} \mathrm{x}$ $\cos ^{2} \mathbf{x}= \pm 1$ $\cos ^{2} \mathrm{x}=+1 \quad \mathrm{x}=\mathrm{O}^{\circ}$ $\cos ^{2} \mathrm{x}=-1 \quad \mathrm{x}=$ imaginário

$\pm \cos 109^{\circ} 28^{\prime} 16^{\prime \prime}=\cos ^{2} x$ $\cos ^{2} \mathrm{x}= \pm 0,3334$ $\cos ^{2} \mathbf{x}=+0,3334$ $\mathrm{x}=54^{\circ} 44^{\prime} 09^{\prime \prime}$ $\cos ^{2} \mathbf{x}=-0,3334$ $\mathrm{x}=$ imaginário

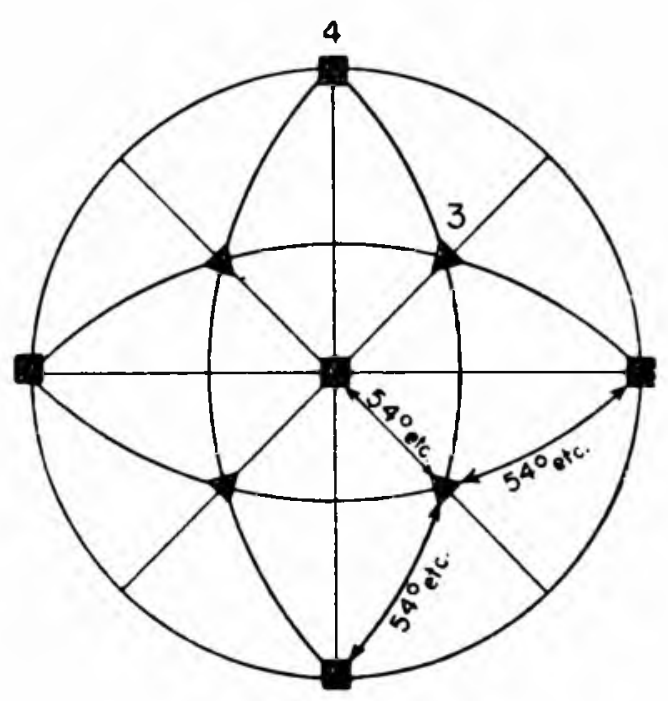

Fig. 12 
Dos resultados obtidos ,apenas dois se mostram viáveis. Porém, quando $\mathrm{x}=0^{\circ}$, embora a solução matemática esteja certa, existe incompatibilidade, pois em tal caso deveria ser admitida a coincidência de $4 \mathrm{com}$ 3, o que constitue um absurdo. O único ângulo possível, portanto, entre um eixo quaternário e um ternário é $54^{\circ} 44^{\prime} 08^{\prime \prime}$ (fig. 12)

Qualquer que seja, por conseguinte a associação entre eixos de simetria imaginada, tal associação poderá ser confirmada ou rejeitada por dedução matemática, aplicando-se simplesmente uma fórmula elemen. tar da trigonometria esférica.

\section{Referências bibliográficas}

1. Camargo, W.G.R. - Associação de eixos ternários na simetria cristalográfica, Ciência e Cultura - vol. 6, n. 3, 1954, pg. 132.

2. HAMmond, J.R. - (1934) - Concise spherical trigonometry.

3. NigGLI, P - (1919) - Geometrische Kristallographie des Deskontinuums.

4. NigGLI, P - (1924) - Lehrbuch der Mineralogie. 